\title{
Hydrogen Absorption in Gd Thin Films
}

\author{
A. Marczyńska, S. Pacanowski, B. Szymański* and L. Smardz \\ Institute of Molecular Physics, Polish Academy of Sciences, M. Smoluchowskiego 17, 60-179 Poznań, Poland

\begin{abstract}
In this contribution we have studied an initial hydrogen absorption in Gd thin films using in-situ X-ray photoelectron spectroscopy (XPS) and ex-situ standard X-ray diffraction. As an initial hydrogen absorption indicator we have used broadening of the Gd- $4 f$ peak. The Gd thin films with a thickness of $200 \mathrm{~nm}$ were deposited at room temperature (RT) using UHV RF magnetron sputtering. As a substrate we have used naturally oxidised $\mathrm{Si}(100)$ wafers with $20 \mathrm{~nm}$ - Pd buffer layer. Furthermore, hydrogen absorption kinetics under a pressure of 100 mbar in $\mathrm{Pd}$ covered $200 \mathrm{~nm}$ Gd thin film was studied at RT using four-point resistivity measurements.
\end{abstract}

DOI: 10.12693/APhysPolA.133.624

PACS/topics: 75.70.-i, 68.55.--a

\section{Introduction}

Rare earth (RE) metals are of great scientific interest because of unusual electronic and magnetic properties which arise from their highly localized $4 f$ electrons. Adsorbates can significantly alter the electronic structure of the underlying substrate and are additionally of great importance in technological processes, e.g., heterogeneous catalysis. RE metals present complex structural and spin configurations when pressure or temperature is changed. Moreover, REs suffer a fast oxidation process when they are exposed to air; a problem that usually reduces their technological applicability. Oxidation problem is enhanced in the case of thin films due to their large surface area. Consequently, studies in RE films are still necessary to better understand their structural and magnetic properties as well as their oxidation process.

Gadolinium is a nearly ideal soft-magnetic material due to the small magnetic anisotropy $[1,2]$. However, one cannot take advantage of its properties because at room temperature (RT) Gd loses the ferromagnetic ordering. On the other hand, it has been recently shown that hydrogenation could enhance the Curie temperature [3].

As the hydrogen concentration in Gadolinium increases, bulk Gd undergoes the following structural transitions [4]: $\alpha$-phase $(h c p, \mathrm{Gd}) / \beta$-phase $\left(f c c, \mathrm{GdH}_{2}\right) / \gamma$ phase $\left(h c p, \mathrm{GdH}_{3}\right)$. Hydrogen-loaded thin films often behave very differently from the corresponding bulk materials $[5,6]$. Contrary to bulk materials which exhibit isotropic lattice expansion when loaded with hydrogen, thin films are clamped to a substrate. The clamping prevents the in-plane lattice expansion and is responsible for in-plane compressive stresses. Therefore, the expansion of hydrogen loaded films is highly anisotropic: the in-plane expansion is suppressed while the out-of-plane expansion is larger than in a corresponding bulk sample [7]. Furthermore, polycrystalline thin films, and especially nanocrystalline films, often exhibit a high den-

*corresponding author; e-mail: szyman@ifmpan.poznan.pl sity of lattice defects. It is known that $\mathrm{H}$ strongly interacts with defects [5]. Initial step of hydride formation in single crystalline gadolinium thin films was studied in Ref. [8-10]. Exposure to low amounts of hydrogen $(1-2 \mathrm{~L})$ on thin films leads to adsorption and as a consequence to a suppression of the surface state [8]. The amount of hydrogen is given in Langmuir $(1 \mathrm{~L}=$ 1 Torr $\times 1 \mathrm{~s})$. Higher amounts $(15 \mathrm{~L})$ of hydrogen result in plastic deformations with induced morphological changes of the surface due to the increased volume of the $\mathrm{GdH}_{2}[8]$.

In this paper we report on structural and electronic properties of Gd thin film deposited by magnetron sputtering. Furthermore, initial hydrogen absorption and absorption kinetics under a pressure of 100 mbar were studied in-situ by X-ray Photoelectron Spectroscopy (XPS) and ex-situ using resistivity measurements, respectively.

\section{Experimental procedure}

$\mathrm{Pd} / \mathrm{Gd}$ bilayer and $\mathrm{Pd} / \mathrm{Gd} / \mathrm{Pd}$ trilayer were prepared at RT using UHV $\left(5 \times 10^{-10}\right.$ mbar $)$ magnetron sputtering [11-13]. In Fig. 1 we show a schematic description of prepared layered structures. As a substrate we have used $\operatorname{Si}(100)$ wafers with naturally oxidised surface to prevent a silicide formation. Therefore we have applied a special heat treatment in UHV before deposition in order to obtain a smooth $\mathrm{SiO}_{2}$ surface layer [14, 15]. The Gd-layers were deposited using a radio frequency $(\mathrm{RF})$ current source. For preparation of the Pd-layers a direct current (DC) source was used. The deposition rates of Gd and Pd were individually checked by quartz thickness monitors. The thickness of individual layers were controlled by varying their deposition times. Furthermore, the calibrations of the deposition rates were also determined by thickness measurements of reference samples using DEC-TAC (Bruker) profilometer and X-ray fluorescence analysis. Finally, a protective layer of $10 \mathrm{~nm}$ $\mathrm{Pd}$ was deposited to allow a fast uptake and release of hydrogen at RT and to avoid oxidation of the Gd layer.

The chemical composition of all the layers was checked in-situ, immediately after deposition, transferring the 


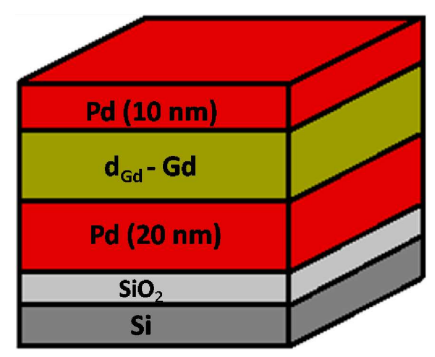

Fig. 1. Schematic description of prepared $\mathrm{Pd} / \mathrm{Gd} / \mathrm{Pd}$ trilayers.

samples to an UHV $\left(4 \times 10^{-11}\right.$ mbar $)$ analysis chamber equipped with XPS. The XPS spectra were measured at RT using a SPECS EA 10 PLUS energy spectrometer with $\mathrm{Al}-\mathrm{K}_{\alpha}$ radiation of $1486.6 \mathrm{eV}$. The energy spectra of the electrons were analysed by a hemispherical analyser $\left(\mathrm{FWHM}_{\mathrm{Mg}-\mathrm{K} \alpha}=0.8 \mathrm{eV}\right.$ for $\left.\mathrm{Ag}-3 \mathrm{~d}_{5 / 2}\right)$. All emission spectra were measured immediately after the sample transfer in vacuum of $8 \times 10^{-11}$ mbar. Details of the XPS measurements can be found in Ref. [16-18].

The structure of the Gd film before and after hydrogenation was examined using standard $\theta-2 \theta$ X-ray diffraction (XRD). Initial step of the hydrogen loading of the $20 \mathrm{~nm} \mathrm{Pd} / 200 \mathrm{~nm}$ Gd bilayer was performed in-situ in the UHV analyse chamber. Moreover, $\mathrm{Pd} / \mathrm{Gd} / \mathrm{Pd}$ trilayer was hydrogenated ex-situ under a pressure of 100 mbar in a HV chamber evacuated to about $10^{-6}$ mbar $[19,20]$. Absorption kinetics was studied using four-point resistivity measurements at RT. Since $\mathrm{Gd}$ is a metal and $\mathrm{GdH}_{3}$ is a semiconductor, the time evolution of hydrogenation can be pursued in real time by monitoring the change of the resistivity. Before hydrogenation the surfaces of the samples were outgased at $450 \mathrm{~K}$ for $1 \mathrm{~h}$. The magnetic characterisation of the $\mathrm{Pd} / \mathrm{Gd} / \mathrm{Pd}$ trilayer before and after hydrogenation was carried out using a Vibrating Sample Magnetometer (VSM) at $295 \mathrm{~K}$ in an in-plane magnetic field up to $2 \mathrm{~T}$.

\section{Results and discussion}

In Fig. 2 we show XPS core-level spectrum of the freshly deposited Gd thin film on $20 \mathrm{~nm}$ Pd buffer layer. Due to well known reactivity of Gd with oxygen we have deposited the thin film after an additional heating of the sample holder and the new substrate at $700 \mathrm{~K}$ for $3 \mathrm{~h}$ and cooling to $295 \mathrm{~K}$. Results showed that after such an outgassing procedure, it is possible to prepare oxygen and carbon free Gd surface. If the outgassing procedure is too short, the sample holder before deposition will not be perfectly clean. Moreover, if the XPS measurements were performed after few hours delay, the sample surface would already be partly oxidised, despite the vacuum of $4 \times 10^{-11}$ mbar in the analysis chamber. In that case the intensity of the XPS signal decreases and the valence band could be artificially broadened. In our case, the oxygen and other surface impurities are practically ab- sent on a such prepared Gd thin film surface. As can be seen in Fig. 2, practically no XPS signal from potential contamination atoms like $\mathrm{O}-1 \mathrm{~s}$ and $\mathrm{C}-1 \mathrm{~s}$ is observed. On the other hand, we have observed oxygen atoms (below 1 at.\%) already adsorbed on Gd surface $2 \mathrm{~h}$ after the preparation. Note that the sample was kept in the analysis chamber in the vacuum of $4 \times 10^{-11}$ mbar.

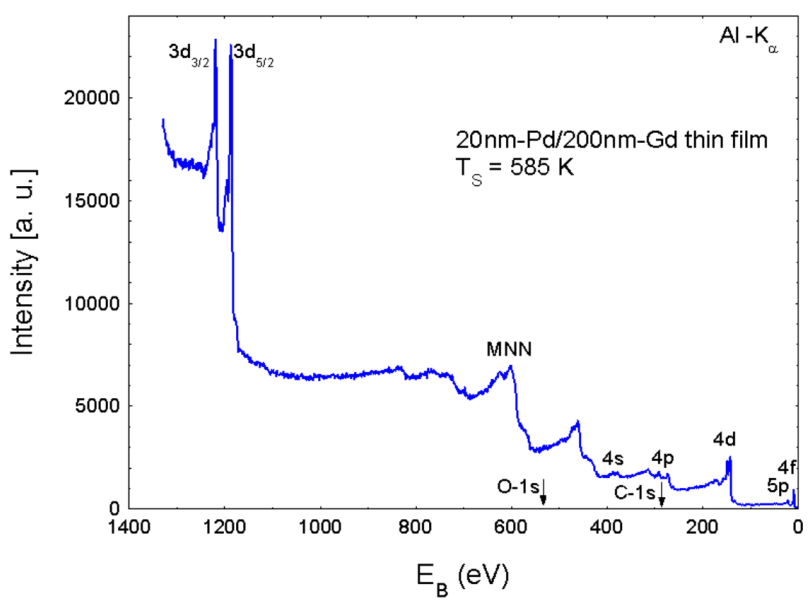

Fig. 2. $\left(\mathrm{Al}-\mathrm{K}_{\alpha}\right)$ XPS spectrum of in-situ prepared $200 \mathrm{~nm}-\mathrm{Gd}$ on $20 \mathrm{~nm}$ - Pd buffer layer at $585 \mathrm{~K}$.

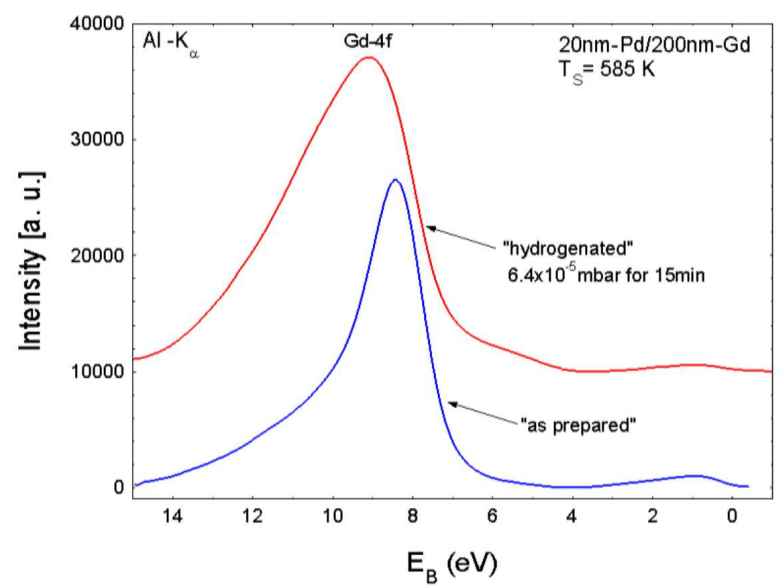

Fig. 3. XPS $\left(\mathrm{Al}-\mathrm{K}_{\alpha}\right)$ valence band spectra of in-situ prepared $200 \mathrm{~nm} \mathrm{Gd}$ on $20 \mathrm{~nm}$ Pd buffer layer. before and after room temperature hydrogenation at a pressure of $6.4 \times 10^{-5} \mathrm{mbar}$ for $15 \mathrm{~min}$.

Fig. 3 shows the XPS valence band for $200 \mathrm{~nm} \mathrm{Gd}$ thin film deposited on $20 \mathrm{~nm} \mathrm{Pd}$ buffer layer before and after hydrogen absorption at a dose of $6.4 \times 10^{-5}$ mbar for $15 \mathrm{~min}$. As can be observed $4 f$ peak is considerably broadened after an initial hydrogen absorption. Moreover, $4 f$ peak is also shifted from 8.4 to $9.15 \mathrm{eV}$. After XPS measurements the $\mathrm{Gd} / \mathrm{Pd}$ bilayer is immediately transferred back to the preparation chamber and the $10 \mathrm{~nm} \mathrm{Pd}$ protective layer was deposited onto hydrogenated $200 \mathrm{~nm}$ Gd thin film. In Fig. 4 we show XRD 


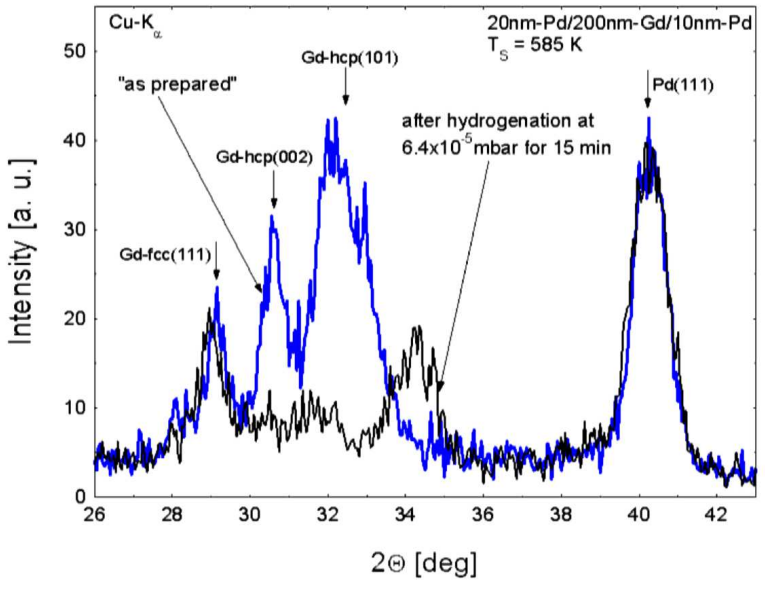

Fig. 4. XRD pattern $\left(\mathrm{Cu}-\mathrm{K}_{\alpha}\right)$ of ,,as prepared" and hydrogenated $20 \mathrm{~nm} \mathrm{Pd} / 200 \mathrm{~nm} \mathrm{Gd} / 10 \mathrm{~nm} \mathrm{Pd}$ trilayers deposited at $585 \mathrm{~K}$.

pattern of the $\mathrm{Gd} / \mathrm{Pd}$ bilayer covered immediately after hydrogenation by $10 \mathrm{~nm}$ Pd protective layer. For a comparison, we also show XRD pattern for the "as prepared" $\mathrm{Pd} / \mathrm{Gd} / \mathrm{Pd}$ trilayer. Note, that the two patterns showed in Fig. 4 are obtained in fact for two different samples deposited under the same preparation conditions. Results showed that the Gd thin film grows in a mixture of $f c c$ and $h c p$ phases [21] as indicated in Fig. 4. After initial step of hydrogenation the Gd hcp peaks practically disappear. On the other hand, in Fig. 4 we have observed a broad (101) peak of mixture of cubic $\mathrm{GdH}_{2}$ and $\mathrm{GdH}_{3}$ phases [22].

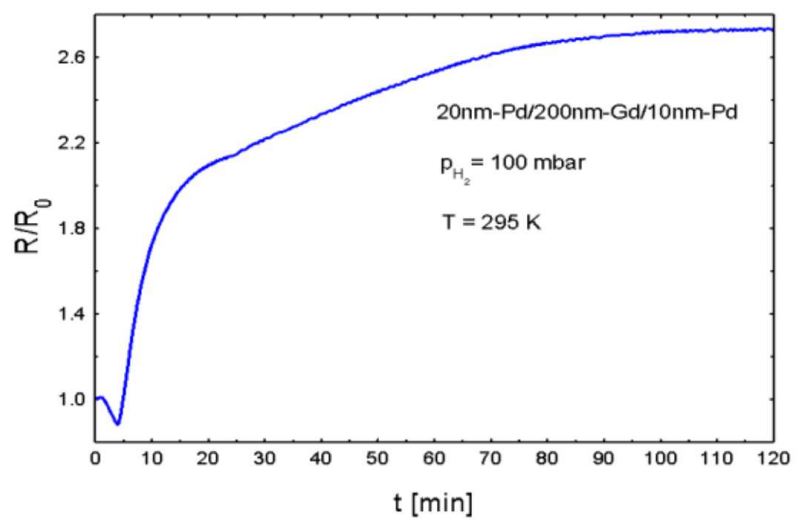

Fig. 5. Relative resistivity $\left(R / R_{0}\right)$ as a function of hydrogenation time under a pressure of 100 mbar. $R_{0}=$ $R(t=0)$.

Magnetic measurements at $295 \mathrm{~K}$ revealed no hysteresis loop for both "as deposited" and hydrogenated $\mathrm{Pd} / \mathrm{Gd} / \mathrm{Pd}$ trilayers. Therefore, we conclude that the $200 \mathrm{~nm}$ Gd thin films are paramagnetic at RT.

Figure 5 shows relative resistivity change of the $20 \mathrm{~nm} \mathrm{Pd} / 200 \mathrm{~nm} \mathrm{Gd} / 10 \mathrm{~nm} \mathrm{Pd}$ alloy thin film dur- ing hydrogen absorption at a pressure of about 100 mbar. Note that immediately after preparation the Gd thin film was covered in-situ by $10 \mathrm{~nm}$ Pd layer to catalyze hydrogen absorption and to protect against oxidation. The sample shows rather slow resistivity change during the first 15 min of RT absorption (see Fig. 5) compared to that measured for nanocrystalline $\mathrm{ZrPd}_{2}$ thin films [23]. Moreover, we have observed a minimum of $R / R_{0}$ after the first $4 \mathrm{~min}$ of the hydrogenation. The above behaviour could be explained by an improve of the $\mathrm{Pd} / \mathrm{Gd}$ and/or $\mathrm{Gd} / \mathrm{Pd}$ interface due to the initial diffusion process of the hydrogen atoms.

In conclusion, results showed that the initial hydrogen absorption in the $200 \mathrm{~nm}$ thick Gd thin film deposited in-situ on $20 \mathrm{~nm}$ Pd buffer layer strongly influences the shape and position of the Gd - $4 f$ peak of the XPS valence band. Moreover, pure Gd thin film practically doesn't desorb hydrogen at RT even under UHV conditions. Therefore, $4 f$ peak could be a very sensitive indicator of the in-situ studies of the hydrogenation mechanism in Gd thin films.

\section{References}

[1] G. Scheunert, C. Ward, W.R. Hendren, A.A. Lapicki, R. Hardeman, M. Mooney, M. Gubbins, R.M. Bowman, J. Phys. D: Appl. Phys. 47, 415005 (2014).

[2] L. Oroszlány, A. Deák, E. Simon, S. Khmelevskyi, L. Szunyogh, Phys. Rev. Lett. 115, 096402 (2015).

[3] E.A. Tereshina, S. Khmelevskyi, G. Politova, T. Kaminskaya, H. Drulis, I.S. Tereshina, Sci. Rep. 6, 22553 (2016).

[4] F.B. Manchester (Ed.), Phase Diagrams of Binary Hydrogen Alloys, ASM International Materials Park, 2000, p. 58.

[5] Q. M. Yang, G. Schmitz, S. Fhler, H. U. Krebs, R. Kirchheim, Phys. Rev. B 54, 9131 (1996).

[6] A. Pundt, R. Kirchheim, Annu. Rev. Mater. Res. 36, 555 (2006).

[7] E. Shalaan, H. Schmitt, K.-H. Ehses, Thin Solid Films 489, 330 (2005).

[8] M. Getzlaff, M. Bode, R. Wiesendanger, Surf. Sci. 410, 189 (1998).

[9] A. Pundt, M. Getzlaff, M. Bode, R. Kirchheim, R. Wiesendanger, Phys. Rev. B 61, 9964 (2000).

[10] S. Wanjelik, O. Stolboushkina, S. Königshofen, M. Getzlaff, J. Alloys Compd. 645, S221 (2015).

[11] L. Smardz, K. Le Dang, H. Niedoba, K. Chrzumnicka, J. Magn. Magn. Mater. 140-144, 569 (1995).

[12] L. Smardz, J. Alloys Comp. 395, 17 (2005).

[13] L. Smardz, K. Smardz, H. Niedoba, J. Magn. Magn. Mater. 220, 175 (2000).

[14] L. Smardz, U. Köbler, W. Zinn, J. Appl. Phys. 71, 5199 (1992).

[15] L. Smardz, U. Köbler, W. Zinn, Vacuum 42, 283 (1991).

[16] L. Smardz, M. Nowak, M. Jurczyk, Int. J. of Hydrogen Energy 37, 3659 (2012). 
[17] L. Smardz, M. Jurczyk, K. Smardz, M. Nowak, M. Makowiecka, I. Okońska, Renewable Energy 33, 201 (2008).

[18] K. Smardz, L. Smardz, I. Okonska, M. Nowak, M. Jurczyk, International Journal of Hydrogen Energy 33, 387 (2008).

[19] J. Skoryna, A. Marczyńska, M. Lewandowski, L. Smardz, J. Alloys Comp. 645, 280 (2015).

[20] J. Skoryna, M. Wachowiak, A. Marczyńska, A. Rogowska,Ł. Majchrzycki, W. Koczorowski, R. Czajka, L. Smardz, Surf. Coat. Techn. 303, 119 (2016).
[21] T.P. Bertelli, E.C. Passamani, C. Larica, V.P. Nascimento, A.Y. Takeuchi, M.S. Pessoa, J. Appl. Phys. 117, 203904 (2015).

[22] E. Shalaan, K.H. Ehses, H. Schmitt, J. Mater. Sci. 41, 7454 (2006).

[23] B. Jabłoński, S. Pacanowski, M. Werwiński, A. Marczyńska, H. Dawczak-Dębicki, A. Szajek, L. Smardz, Acta Phys. Pol. A 133, 620 (2018). 